\title{
Evidence of a Neuroprotective Function for Niclosamide in Human SH-SY5Y Neuroblastoma and Rat PC12 Neural Cells
}

\section{Kevin C Bermea ${ }^{1}$, Edgar A Casillas ${ }^{2}$, Liza D Morales ${ }^{3}$, Laura L Valdez ${ }^{1}$, Brenda Bin $\mathrm{Su}^{1}$, Andrew Tsin ${ }^{1}$ and Benxu Cheng ${ }^{1 *}$}

${ }^{1}$ Department of Molecular Science, School of Medicine, The University of Texas Rio Grande Valley, United States of America

${ }^{2}$ Department of Physical Therapy, School of Health Professions, The University of Texas Health Science Center at San Antonio, United States of America ${ }^{3}$ Department of Human Genetics and the South Texas Diabetes and Obesity Institute, School of Medicine, The University of Texas Rio Grande Valley, United States of America *Corresponding Author: Benxu Cheng, Department of Molecular Science, School of Medicine, The University of Texas Rio Grande Valley, United States of America.
Received: June 24, 2020

Published: August 31, 2020

(C) All rights are reserved by Benxu Cheng., et al.

\begin{abstract}
Neurodegenerative disease is a debilitating and incurable condition that affects millions. It results in the loss of function and eventual death of neural cells. It has been established that exposure of model neuronal-derived cell lines to the proteasome inhibitor MG132 emulates in vitro neurodegeneration as evidenced by a marked decrease in protein degradation concomitant with an increase in aggregate intracellular ubiquitinated proteins and endoplasmic reticulum (ER) stress (via upregulation of CHOP/GADD153). Consequently, apoptosis ensues corresponding to the upregulation of p53, activation of caspase-3, cleavage of poly (ADP-ribose) polymerase (PARP), and DNA condensation/fragmentation. In this study, we investigated the potential neuroprotective function of niclosamide and its associated signaling mechanisms in SH-SY5Y and PC12 neural cells exposed to MG132. All markers of MG132induced neurodegeneration, including the accumulation of ubiquitinated proteins, were prevented by exposure to niclosamide. In addition, niclosamide was shown to induce autophagy independently and to enhance autophagy induced by MG132. These results show that niclosamide may serve as a potential neuroprotective agent through its ability to inhibit proteasome dysfunction-induced protein ubiquitination.
\end{abstract}

Keywords: Neurodegeneration; Proteasome Dysfunction; Protein Ubiquitination; ER Stress; Apoptosis; Autophagy; Niclosamide; Neuroprotection

\section{Abbreviations}

PD: Parkinson's Disease; UPS: Ubiquitin-Proteasome System; ER: Endoplasmic Reticulum; UPR: Unfolded Protein Response; FBS: Fetal Bovine Serum; PFA: Paraformaldehyde; LC3-I/LC3-II: Microtubule-Associated Protein Light Chain 3.

\section{Introduction}

Parkinson's disease (PD) is a progressive neurodegenerative disease that affects motor control. It was estimated that in the year 2010, PD affected approximately 680,000 individuals aged 45 years and older in the US, and it was projected to increase to over 1 million by the year 2030 [1]. PD is characterized by a loss of dopaminergic neurons of the substantia nigra pars compacta and by the presence of intracytoplasmic proteinaceous deposits known as Lewy bodies [2].

Intracellular protein degradation is well regulated by the ubiquitin-proteasome system (UPS) and the autophagy-lysosome system [3]. Proteasomes are protein complexes containing highly 
conserved multi-catalytic proteases, found in the nucleus and cytoplasm of eukaryotes, which breakdown proteins that have been inactivated by ubiquitination. Lewy bodies contain multiple UPS components, such as ubiquitin and proteasome subunits [4]. In the case of PD, Lewy bodies also consist of highly ubiquitinated protein aggregates in degenerated dopaminergic neurons [5]. Most neurodegenerative diseases like PD are characterized by the presence of ubiquitin-positive aggregates [6], indicating that the UPS plays a critical role in the decay of these neurons in the setting of PD. Ample evidence suggests that defects in the UPS contribute to the development of PD. Post-mortem biopsies have shown decreased proteasome activity in the substantia nigra pars compacta [7]. Proteasome activity has been shown to also decrease with normal aging, indicating loss of proteasome activity may be a factor in the elderly being vulnerable to acquiring a neurodegenerative disorder like PD [8]. Therefore, identifying therapeutic agents capable of targeting protein ubiquitination induced by proteasome dysfunction, and the accumulation of cytoplasmic protein aggregates may be an effective strategy for developing novel treatments against PD.

Autophagy has beneficial, pro-survival effects on cells, especially neuronal cells, which are particularly vulnerable to the accumulation of protein deposits; therefore, the effective degradation of protein aggregates is essential for age-dependent maintenance of neuronal viability. It has been described that autophagy is a cellular response to extracellular and intracellular stress conditions $[9,10]$. Most neurodegenerative diseases are marked not only by the presence of ubiquitin-positive protein aggregates, but also by the presence of autophagy abnormalities [11]. Since aggresome (an aggregation of misfolded proteins) formation is essential for activation of protein degradation via autophagy, it is thought that this process could be an effective therapeutic target for neurodegenerative diseases such, as Alzheimer's disease, Parkinson's disease, and Huntington's disease [12-15].

Proteasome inhibition has been widely used to study the UPS in neurodegenerative conditions [16,17]. The mechanisms of cytotoxicity induced by proteasome inhibition are very complex and likely cell-specific. Proteasome inhibition leads to the accumulation of misfolded proteins in the endoplasmic reticulum (ER) and results in ER stress in neuronal cells [18-20]. In addition, proteasome inhibition is able to induce activation of an ER stress-me- diated apoptosis pathway [21]. Neuronal cell death is triggered by complex, specific-cell signaling known as the Unfolded Protein Response (UPR) [20].

In our previous studies, we demonstrated that IGF- 1 and retinoic acid protect against proteasome inhibition-induced cytotoxicity by stimulating the PI3/AKT signaling pathway. However, the levels of ubiquitinated proteins between protected and non-protected cells were not significantly different $[22,23]$. Very interestingly, the anti-helminthic niclosamide demonstrated an ability to reduce the accumulation of poly-ubiquitinated proteins induced by MG132mediated proteasome inhibition [24], although, the mechanism of the action remains unclear. Niclosamide is an FDA approved drug used to treat parasitic worm infections, which has been shown to influence multiple cell processes and has been proposed as a potential therapeutic agent for many conditions. For instance, it has been proposed as a potential anti-tumoral agent due to its ability to target multiple growth signaling pathways such as Wnt [25] and STAT3 signaling [26-28]. Niclosamide also has been shown to reduce diabetic symptoms in mouse models and it impeded diabetic kidney disease progression by reducing renal hypertrophy and podocyte dysfunction and by suppressing renal cortical activation of the mTOR/4E-BP1 signaling pathway [29,30]. Interestingly, another recent study showed niclosamide provided neuroprotection against oxaliplatin-induced oxidative stress and neuroinflammation which lead to neurological dysfunction [31].

Niclosamide was shown to inhibit the formation of large ubiquitin-containing aggregates induced by the proteasome inhibitor MG132 [24] and to promote autophagy [32]. Therefore, we hypothesized that niclosamide could have neuroprotective properties through its ability to suppress proteasome inhibition-induced protein ubiquitination, apoptosis, ER stress and other types of cell death, like autophagy. In the current study, we demonstrate niclosamide markedly reduces protein ubiquitination in human SHSY5Y neuroblastoma and rat PC12 neural cells induced by MG132 treatment in a dose-dependent manner and it suppresses MG132induced apoptosis by inhibiting the upregulation of p53 and ER stress. These results suggest that niclosamide may render current treatments of neurodegenerative diseases, like PD, more effective by protecting neuronal cells against proteasome dysfunction which triggers the aggregation of ubiquitinated proteins, and subsequently, cell death. 


\section{Materials and Methods}

\section{Chemicals}

Proteasome inhibitor MG132 and niclosamide were purchased from Sigma-Aldrich (St. Louis, MO). Both MG132 and niclosamide were dissolved in DMSO stored at $-20^{\circ} \mathrm{C}$. Hoechst 33342 was obtained from Life Technologies (Eugene, OR, USA).

\section{Cell culture}

Human SH-SY5Y cells and rat PC12 pheochromocytoma cells were purchased from the American Type Culture Collection (Manassas, VA, USA). The SH-SY5Y cells were cultured at 5\% CO2 at $37^{\circ} \mathrm{C}$ in EMEM: Ham's F-12K medium (1:1) and PC12 cells were containing $10 \%$ fetal bovine serum (FBS), and 5\% horse serum with penicillin (100 units $/ \mathrm{mL}$ ), and streptomycin $(100 \mu \mathrm{g} / \mathrm{mL})$. The cells were sub-cultured weekly in $60 \mathrm{~mm}$ or $100 \mathrm{~mm}$ cell culture dishes and used for experiments at $85-90 \%$ confluence of the cell monolayer. For Hoechst 33342 nucleic acid staining, cells were grown in 4-well chamber slides.

\section{Cell treatment}

The proteasome inhibitor MG132 was used to induce cytotoxicity in SH-SY5Y and PC12 cultures. DMSO was used as a control vehicle, with a final concentration of less than $0.1 \%$ in the cell culture medium. Cells were treated either with MG132 or niclosamide alone or in combination for various times as needed.

\section{DNA damage assay}

DNA damage and chromatin condensation were detected using Hoechst staining of cells cultured in glass bottom dishes. Briefly, after treatment cells were fixed with $3.7 \%$ paraformaldehyde (PFA), washed with PBS, and stained with Hoechst 33342 (5 $\mu \mathrm{g} /$ $\mathrm{ml}$ ) solution (Sigma St. Louis, MO, USA). Stained cells were then mounted and imaged with a confocal microscope.

\section{Western blot analysis}

Changes in protein expression were measured via Western blot analysis. Protein homogenates were prepared as follows: the cells were lysed in ice-cold RIPA lysis buffer containing protease and phosphatase inhibitor cocktails (Santa Cruz Biotechnology, Santa Cruz, CA, USA). Clear lysates were obtained by centrifugation at $4^{\circ} \mathrm{C}$ for $20 \mathrm{~min}$ at $13,000 \mathrm{rpm}$ in a refrigerated microcentrifuge. Protein concentrations were determined using the Pierce BCA
Protein Assay Kit (Pierce, Rockford, IL, USA) following the manufacturer's instructions. Equal amounts of the protein samples (25-30 $\mu \mathrm{g}$ ) were separated using a $10 \%$ or $4-20 \%$ gradient polyacrylamide gel (Bio-Rad Laboratories, Hercules, CA, USA), transferred to nitrocellulose membranes, and blocked for either $1 \mathrm{~h}$ at room temperature or overnight at $4^{\circ} \mathrm{C}$ with Tris buffer containing $0.1 \%$ Tween 20 (TBST, pH 7.4) and 5\% (w/v) nonfat dried milk. The blotted membranes were incubated with specific primary antibodies for $1 \mathrm{~h}$ at room temperature or overnight at $4^{\circ} \mathrm{C}$. Antibodies against Ubiquitin, cyclin D, and survivin were purchased from Santa Cruz Biotechnology (Dallas, TX, USA) while CHOP, LC3, Cleaved PARP, and cleaved caspase-3 antibodies were purchased from Cell Signaling (Beverly, MA, USA). Antibody to ß-actin was obtained from Sigma (St. Louis, MO, USA). The membranes were washed and incubated for $1 \mathrm{~h}$ with appropriate horseradish peroxidase-conjugated secondary antibodies. The protein bands were detected using a chemiluminescent method (ECL), according to the manufacturer's instructions, and band densities analyzed by ImageJ software.

\section{Results and Discussion}

Niclosamide inhibits MG132-induced protein ubiquitination in SH-SY5Y cells and PC12 cells

Previously, we found niclosamide promoted protein ubiquitination in human glioblastoma cells [33]. However, recent work demonstrated niclosamide can block protein ubiquitination caused by proteasome inhibition in human SH-SY5Y cells [24]. To confirm this effect of niclosamide, SH-SY5Y cells were treated with different concentrations of niclosamide in the presence or absence of $5 \mu \mathrm{M}$ MG132 for $24 \mathrm{~h}$. The cell lysates were prepared for Western blot assays using anti-ubiquitin antibody. As shown in Figure 1, Western blot analysis confirmed MG132 induces a large increase in ubiquitinated protein levels, especially at higher molecular weights. However, protein ubiquitination in MG132-treated cells was clearly suppressed with the addition of niclosamide at a concentration greater than or equal to $0.5 \mu \mathrm{M}$. We also examined the effect of niclosamide in rat PC12 neural cells. As shown in Figure 2, similar to SH-SY5Y cells, incubation with MG132 alone promoted protein ubiquitination, while co-incubation of MG132 with niclosamide inhibited ubiquitination in a dose-dependent manner compared to MG132only treatment. These results further support opposing effects of MG132-induced protein ubiquitination in different neuronal cell types. 


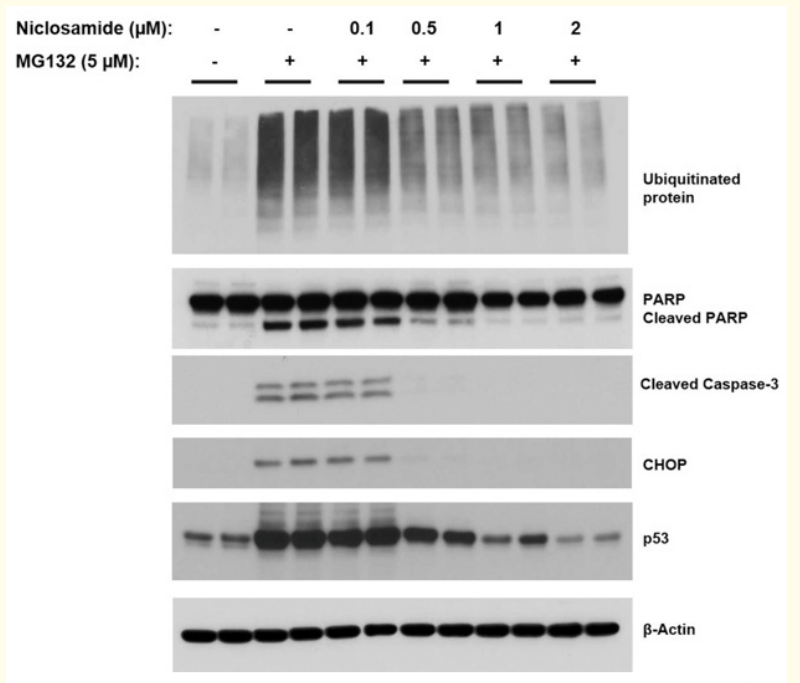

Figure 1: Effect of niclosamide on the cell response to protesome inhibition in SH-SY5Y cell line. SH-SY5Y cells were treated with 5 $\mu \mathrm{M}$ MG132 in the presence or absence of an increasing concentration of niclosamide for $24 \mathrm{~h}$. Western blot was performed using antibodies against p53, CHOP, ubiquitin, PARP, cleaved PARP, cleaved caspase 3 and $\beta$-Actin.

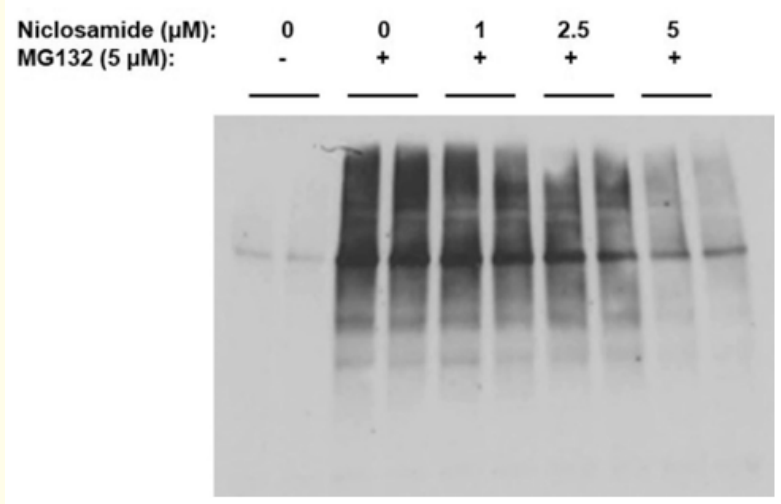

Figure 2: Effect of niclosamide on protein ubiquitination within PC12 cell line. PC12 cells were treated with $5 \mu$ M of MG132 in presence or absence of an increasing concentration of niclosamide for $24 \mathrm{~h}$. Western blot was performed with antibody against ubiquitin.

\section{Niclosamide inhibits MG132-induced apoptosis}

Neurodegenerative diseases like PD result in the death of neural cells. The aggregation of ubiquitinated proteins caused by proteasomal inhibition can trigger cell death or apoptosis. Given that niclosamide can inhibit protein ubiquitination, we aimed to determine whether this compound could also inhibit ubiquitinationinduced apoptosis. SH-SY5Y cells were treated with $5 \mu$ M MG132 in the presence or absence of $1 \mu \mathrm{M}$ niclosamide for $24 \mathrm{~h}$. Cell lysates were then prepared for Western blot probed with antibodies against cleaved caspase- 3 and PARP (poly ADP ribose polymerase), which are well-known markers of apoptosis. As shown in Figure 3, treatment with $5 \mu \mathrm{M}$ MG132 for $24 \mathrm{~h}$ resulted in a clear increase in caspase- 3 and PARP cleavage as compared to the non-treated control. Incubation with niclosamide alone had no effect on the levels of PARP and caspase-3 cleavage; however, the combination of niclosamide with MG132 resulted in a marked reduction of caspase-3 and PARP cleavage in comparison to the MG132-only treatment. In addition, the same experiment was performed in the PC 12 cell line, and as expected, MG132 treatment triggered caspase- 3 and PARP cleavage (Figure 4). Similarly, caspase-3 cleavage and PARP cleavage were blocked by treatment with niclosamide. Thus, these results indicate that niclosamide suppresses proteasome inhibition induced apoptosis in neuronal cells. Moreover, they suggest niclosamide may protect against the effects of neurodegeneration.

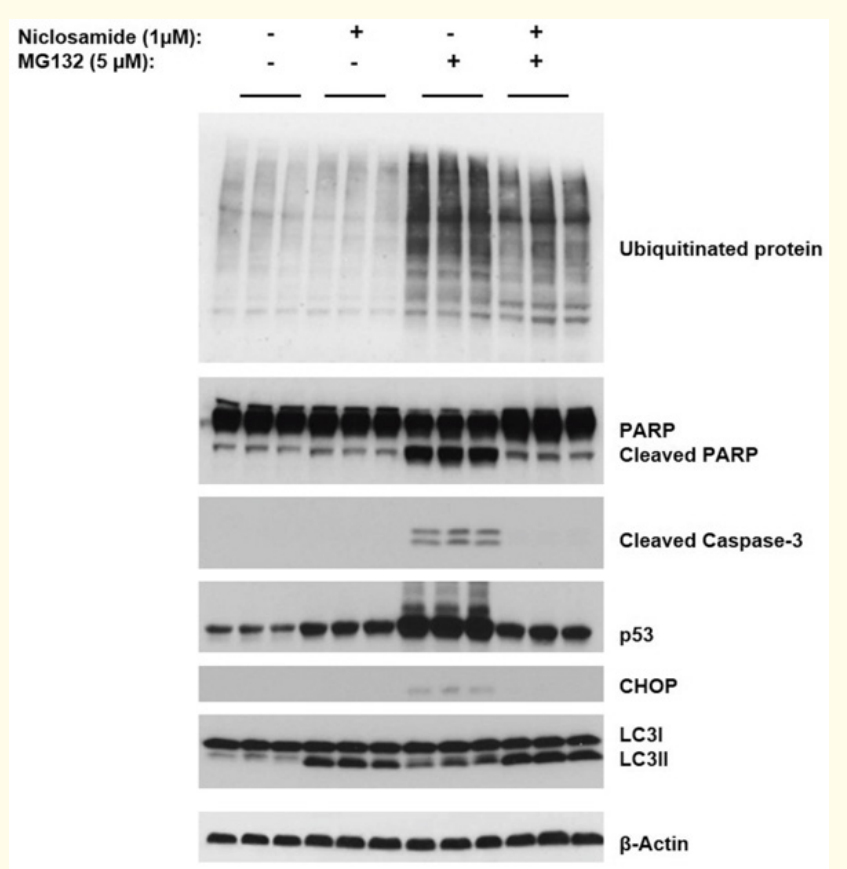

Figure 3: Effect of niclosamide on apoptosis, ER Stress and autophagy in SH-SY5Y cells under conditions of proteasome inhibition. SH-SY5Y cells were treated with $1 \mu \mathrm{M}$ niclosamide in the presence or absence of $5 \mu \mathrm{M}$ MG132 for $24 \mathrm{hrs}$. Western blot was performed with antibodies against p53, LC3I, LC3II, $\beta$-Actin, cleaved caspase-3, PARP, cleaved PARP, and ubiquitin. 


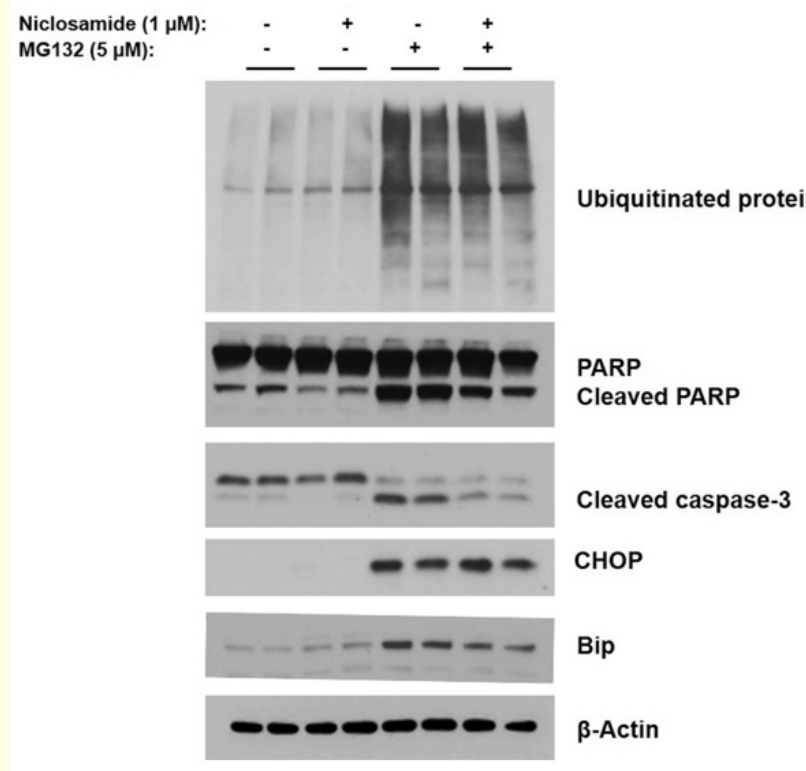

Figure 4: EEffect of niclosamide on apoptosis and ER stress in PC12 cells under conditions of proteasome inhibition. PC12 cells were treated with $1 \mu \mathrm{M}$ niclosamide in the presence or absence of 5 MM MG132 for $24 \mathrm{~h}$. Western blot was performed with antibodies against ubiquitin, PARP, caspase-3, CHOP, Bip and $\beta$-actin.

Nuclear condensation and DNA fragmentation are characteristics of cells undergoing apoptosis, and it has been reported that MG132 treatment is able to trigger these apoptotic events [34]. To confirm the inhibitory effect of niclosamide on MG132-induced apoptosis, SH-SY5Y cells were treated with $10 \mu \mathrm{M}$ MG132 in the presence or absence of $1 \mu \mathrm{M}$ niclosamide for $24 \mathrm{~h}$. Then, cells were fixed and stained with nuclear dye Hoechst 33243. As shown in Figure 5, little to no nuclear condensation was observed in DMSOtreated control cells, while treatment with MG132 only caused extensive nuclear condensation, as evidenced by nuclear shrinkage. As expected, little to no nuclear condensation was observed in the cells treated with niclosamide or with niclosamide/MG132 co-treatment. The data showed niclosamide can inhibit MG132induced DNA fragmentation and therefore, apoptosis. It should be noted that cells treated with niclosamide displayed an abnormal morphology and reduced proliferation rate, as compared to control cells. However, like the control, no detached cells were noted after exposure to $1 \mu \mathrm{M}$ niclosamide for $24 \mathrm{~h}$, implying that although niclosamide caused some type of cellular stress, the cells did not undergo cell death. Further investigation to identify the cause of the abnormal morphology will be needed.
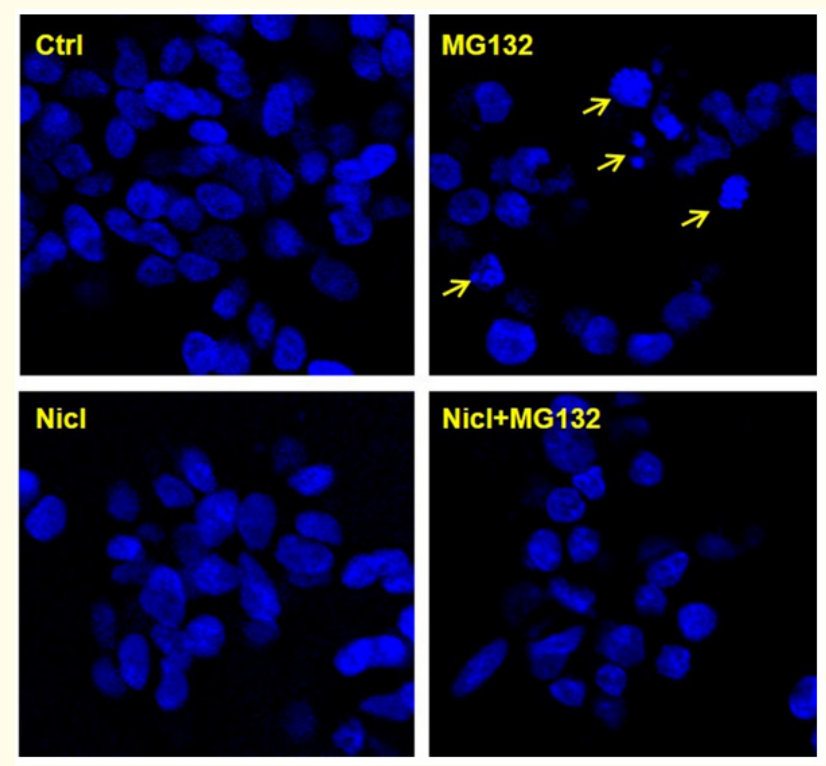

Figure 5: Niclosamide represses MG132-induced DNA condensation/fragmentation in SH-SY5Y cells. SH-SY5Y cells were treated with $5 \mu \mathrm{M}$ niclosamide and $10 \mu \mathrm{M}$ MG132 for 24 hours. Cells were fixed and stained by Hoechst 33258. (Preference for color: print and online).

Niclosamide attenuates MG132-mediated p53 expression in SH-SY5Y cells

The transcription factor p53 is a well-known tumor suppressor protein, and it is a critical regulator of apoptosis. It is known that MG132 upregulates p53 expression, therefore, we examined the effect of niclosamide on MG132-mediated p53 expression. SH-SY5Y cells were exposed to $5 \mu \mathrm{M}$ MG132, $1 \mu \mathrm{M}$ niclosamide for $24 \mathrm{~h}$, and then total protein lysates were prepared for Western blot analysis with anti-p53 antibody. As expected, proteasome inhibitor MG132 markedly induced p53 expression, whereas co-treatment with niclosamide completely reversed this effect (Figure 3). The data implies niclosamide suppresses apoptosis triggered by proteasome inhibition by inhibiting $\mathrm{p} 53$ activation.

Niclosamide inhibits ER stress and pro-apoptotic UPR-associated signaling

Accumulation of unfolded or misfolded proteins in the ER can trigger ER stress-mediated apoptosis $[35,36]$. As previously men- 
tioned, the UPR is activated in response to ER stress to remove misfolded or unfolded proteins in the ER and re-establish cellular homeostasis. If the UPR is unable to clear these proteins, then apoptosis is initiated. Previous studies have shown that proteasome inhibition by MG132 was able to induce ER stress through the upregulation of the transcription factor CHOP (CCAAT/Enhancer-Binding Protein Homologous Protein) and the chaperone BiP (Binding immunoglobulin protein), which are both markers of ER stress [34]. To evaluate the effect of niclosamide on MG132induced ER stress, we treated SH-SY5Y or PC12 cells with MG132, niclosamide, or both for $24 \mathrm{~h}$. Incubation with MG132 alone resulted in a strong increase in CHOP expression in both cell lines, which correlated with an increase in caspase-3 and PARP cleavage (Figures 3, 4 and 6), indicating stimulation of ER stress also promoted apoptosis. Niclosamide alone had no effect on ER stress or apoptosis, as compared with untreated controls and MG132-only treatment in either cell line. However, co-treatment of MG132 with niclosamide dramatically decreased the expression of CHOP in $\mathrm{SH}$ SY5Y cells (Figures 3 and 6). On the other hand, niclosamide had no effect on MG132-mediated upregulation of CHOP expression in PC12 cells; instead, we found that niclosamide reduced MG132 induction of BiP expression, which is another downstream target of UPR (Figure 4). Niclosamide had no effect on BiP expression levels in SH-SY5Y cells (Figure 6). These results imply niclosamide may inhibit ER stress and associated UPR-mediated apoptotic signaling by different mechanisms depending on cell type (Figure 6).

\section{Niclosamide activates autophagy}

As previously mentioned, along with the UPS, autophagy is a mechanism by which misfolded proteins can be removed. Autophagy is also known to be an alternative mechanism of cell death, and dysregulation of this process during neurodegeneration can contribute to neuron cell death. Studies have demonstrated that impairment in the UPS can induce autophagy, and loss of autophagy in mouse brains results in neurodegeneration concomitant with an increase in protein ubiquitination [37]. Proteasome inhibition by MG132 has been shown to induce autophagy in SH-SY5Y cells [38]. To investigate the role of autophagy in MG132-induced apoptosis and the effect of niclosamide on these mechanisms, SH-SY5Y cells and retinoic acid (RA)-differentiated SH-SY5Y cells were treated with niclosamide, MG132, or both for $24 \mathrm{~h}$ then, cell lysates were prepared for Western blot to evaluate the conversion of the microtubule-associated protein light chain 3 I (LC3I) to the microtubule-associated protein light chain 3 II (LC3-II), as its presence is characteristic of autophagy. After exposure of cells to MG132 for $24 \mathrm{~h}$, an increase in the conversion of LC3-I to LC3-II was observed compared to untreated control in both cell types. Interestingly, niclosamide-only treatment also appeared to enhance LC3-II conversion, particularly in RA-differentiated SHSY5Y cells (Fig 7). Treatment of undifferentiated SH-SY5Y cells with both niclosamide and MG132 seemed to have a synergistic effect and promoted autophagy to a level greater than treatment with either niclosamide or MG132 alone. However, the same effect was not observed in RA-differentiated cells. The data implicated that proteasome inhibition can promote apoptosis via autophagy, however, niclosamide does not block MG132-induced ER-stress and cell death through its effect on autophagy. To confirm this hypothesis, SH-SY5Y cells were pretreated with the autophagy inhibitor 3-MA before exposure to MG132 and niclosamide. Pretreatment with 3-MA did not show any significant difference in the niclosamide effect, suggesting that although the promotion of autophagy may contribute to the protective effect of niclosamide, other mechanisms might be involved (Figure 6).

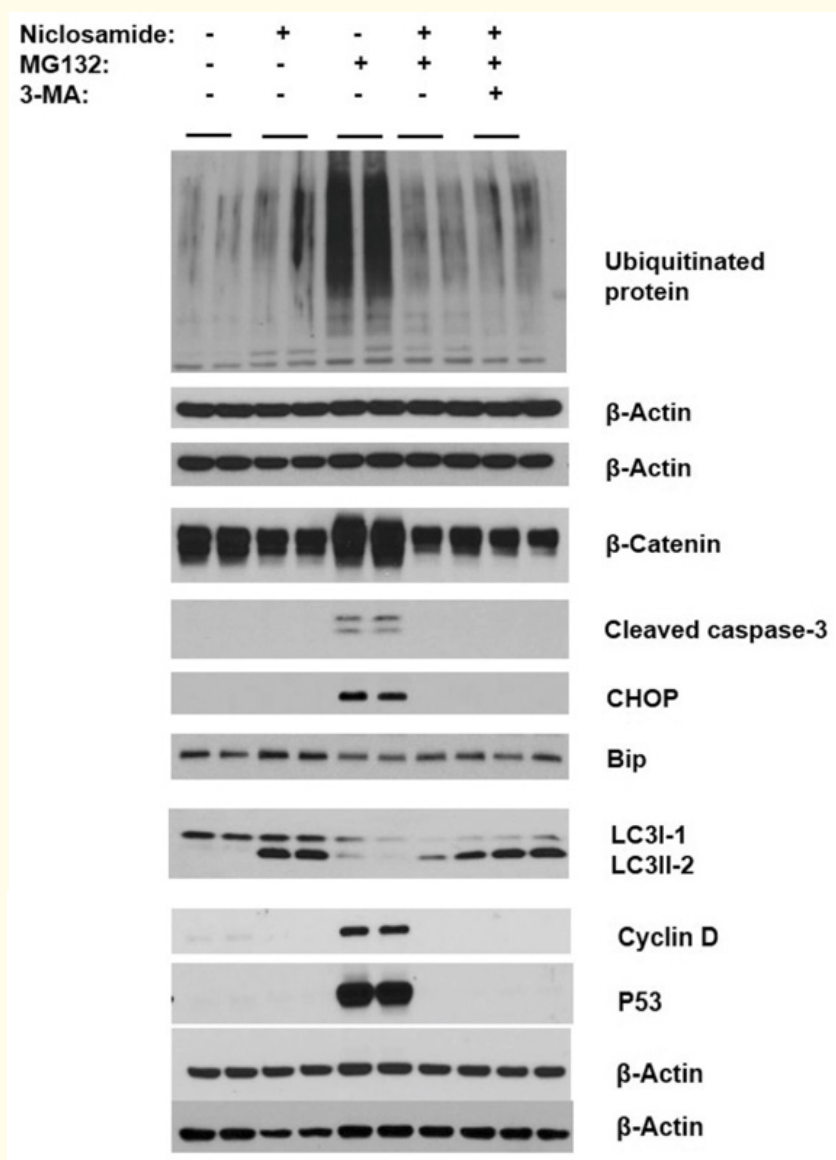

Figure 6: Effect of niclosamide on MG132-induced ER stress. SH-SY5Y cells were treated with niclosamide in the presence or absence of MG132 or 3-MA. Western blot was performed with antibodies against cleaved caspase-3, CHOP, BiP, LC3I-1, LC3II-2, P53, cyclin D, PARP, and cleaved PARP levels. 

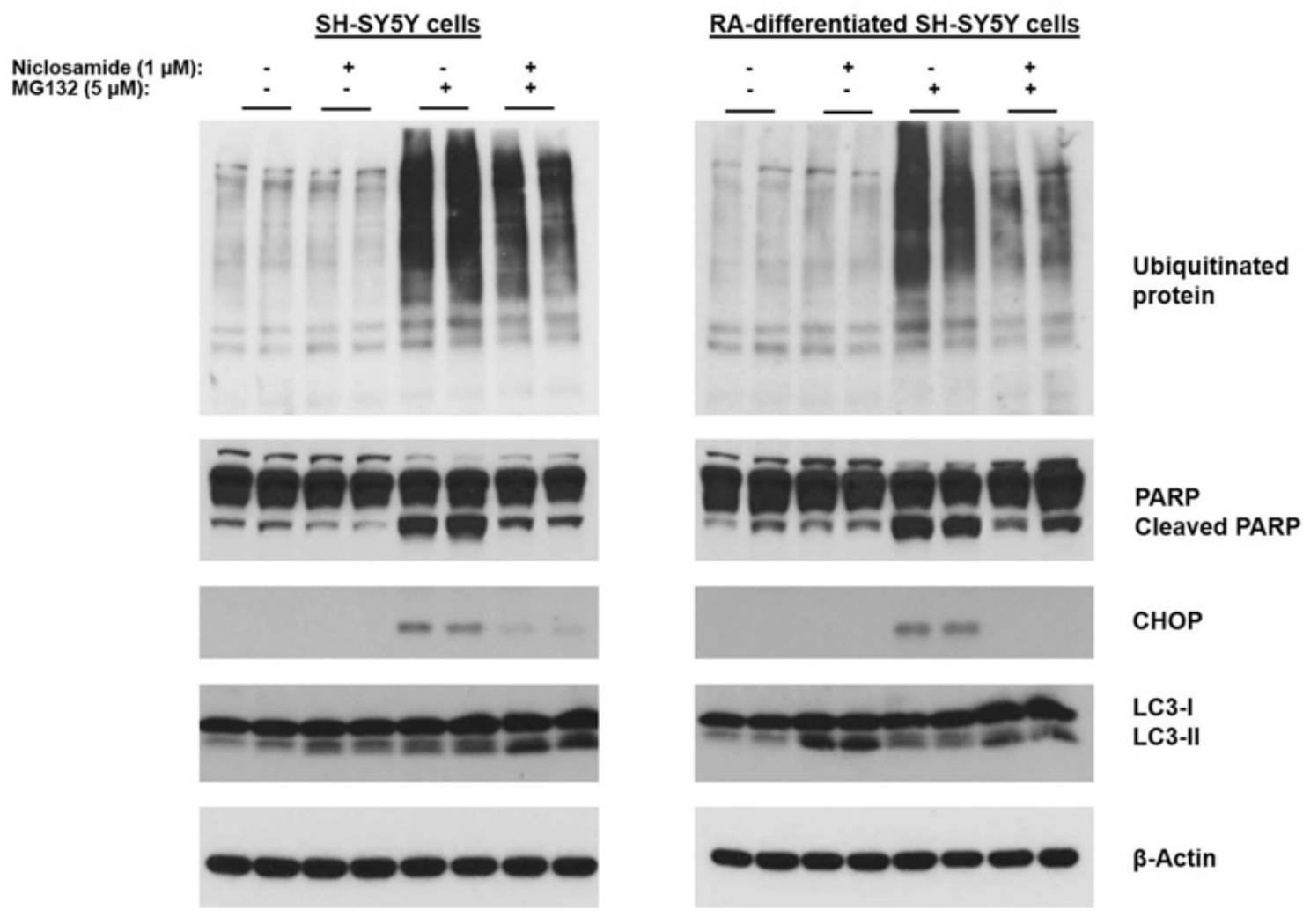

Figure 7: Effect of niclosamide on MG132-induced autophagy. Undifferentiated and differentiated SH-SY5Y cells were treated with 1 $\mu \mathrm{M}$ niclosamide in the presence or absence of $5 \mu \mathrm{M}$ MG132 for 24h. Western blot was performed with antibodies against ubiquitin (Ub), PARP, cleaved PARP, CHOP, LC3I, LC3II and $\beta$-Actin.

\section{Discussion}

Dysfunction of the proteasome can lead to the accumulation of misfolded proteins, which triggers endoplasmic reticulum stress and induces autophagy [39]. In the present study, we report that niclosamide can rescue human SH-SY5Y and rat PC12 neural cells from ER stress caused by proteasome inhibition through its ability to inhibit protein ubiquitination. Exposure to MG132 results in the induction of ER stress and UPR, and subsequently cell death if cell homeostasis cannot be restored by the UPR. Accumulation of misfolded proteins in the ER results in activation of proteins such as CHOP by the UPR and CHOP expression can promote apoptosis [40]. Therefore, inhibition of CHOP expression is one mechanism by which niclosamide could reduce apoptosis induced by proteasome inhibition in neural cells. Niclosamide effectively reduced the expression of CHOP in SH-SY5Y cells. However, it failed to suppress MG132-mediated upregulation of CHOP in PC12 cells, although there was a remarkable decrease in activation of caspase 3 and the subsequent PARP cleavage. These findings suggest proteasome inhibition triggers different UPR-associated apoptotic pathways - CHOP-dependent and CHOP-independent pathways - depending on the cell type, but niclosamide can act on both to promote neural cell survival.

PARP is cleaved by caspase- 3 and this cleavage neutralizes the ability of PARP to participate in DNA repair, resulting in apoptosis [41]. MG132 induces apoptosis, as demonstrated by the activation of caspase- 3 and PARP cleavage, nucleic condensation, and DNA fragmentation [34]. In this study, we showed that niclosamide inhibits the activation of caspase- 3 and thus prevents PARP cleavage, implying niclosamide can negatively regulate apoptosis caused by 
proteasome inhibition through interference in the caspase signaling cascade.

Autophagy is the primary mechanism of degrading and recycling long-lived proteins and damaged organelles and consequently, it can function as a compensatory mechanism to degrade damaged, misfolded or dysfunctional cytoplasmic proteins when the proteasome is inhibited [42]. In fact, defects in autophagy are found to be associated with neuronal loss in neurodegenerative diseases, because abnormal proteins and damaged organelles could not be cleared from the neurons [43]. The function of autophagy in cells and in the development of human disease has been investigated extensively, and various studies have revealed that autophagy can function as either a pro-survival mechanism or as an alternative cell death signaling pathway, depending on cellular state and context $[44,45]$. In the case of neurodegenerative diseases, autophagy can exert a protective effect against neuronal injury by preventing accumulation of cytotoxic factors, such as misfolded proteins [46]. On the other hand, autophagy-induced cell death has been described to occur in association with several neurodegenerative disorders, including PD and $\mathrm{AD}[47,48]$ and it has been suggested that constitutive activation of autophagy may directly lead to neuronal cell death [49]. Our findings revealed exposure to niclosamide resulted in an increase in autophagy which implied neural protection from protein ubiquitination by promoting autophagy for the removal of these proteins. More studies are required to identify the mechanism of action of niclosamide.

\section{Conclusion}

This is the first report showing that niclosamide can inhibit apoptosis induced by proteasome inhibition in both SH-SY5Y and PC12 neuronal cells. In fact, we found that even a low concentration of niclosamide $(0.5 \mu \mathrm{M})$ was able to repress MG132-induced ubiquitination. The findings show that autophagy and ER-stress, which are involved in the neurotoxicity of proteasome inhibition and the associated molecular mechanisms, can be regulated by niclosamide. Therefore, the use of niclosamide in conjunction with current treatments of neurodegenerative disorders may be a valid strategy for prolonging neuronal survival in cells undergoing cytotoxic stress due to the aggregation of ubiquitinated proteins as a result of proteasome dysfunction.

\section{Acknowledgements}

This work is supported by the Department of Molecular Science, School of Medicine, The University of Texas Rio Grande Valley and funding from the State of Texas Innovative Research and Development Program.

\section{Conflict of Interest}

No financial interest or conflict of interest exists.

\section{Bibliography}

1. Marras C., et al. "Prevalence of Parkinson's disease across North America”. NPJ Parkinson's Disease 4 (2018): 21-21.

2. Martínez-Banaclocha MA. "N-acetyl-cysteine in the treatment of Parkinson's disease. What are we waiting for?" Medical Hypotheses 79.1 (2012): 8-12

3. Nedelsky NB., et al. "Autophagy and the ubiquitin-proteasome system: collaborators in neuroprotection". Biochimica et Biophysica Acta 1782.12 (2008): 691-699.

4. Lennox, G., et al. "Anti-ubiquitin immunocytochemistry is more sensitive than conventional techniques in the detection of diffuse Lewy body disease". Journal of Neurology, Neurosurgery, and Psychiatry 52.1 (1989): 67-71.

5. Chung KKK., et al. "Parkin ubiquitinates the [alpha]-synucleininteracting protein, synphilin-1: implications for Lewy-body formation in Parkinson disease". Nature Medicine 7.10 (2001): 1144-1150.

6. Ardley HC and PA Robinson. "The Role of Ubiquitin-Protein Ligases in Neurodegenerative Disease". Neurodegenerative Diseases 1 (2004): 71-87.

7. Tofaris GK., et al. "Ubiquitination of alpha-synuclein in Lewy bodies is a pathological event not associated with impairment of proteasome function". Journal of Biological Chemistry 278.45 (2003): 44405-44411.

8. Friguet B., et al. "Protein Degradation by the Proteasome and Its Implications in Aging". Annals of the New York Academy of Sciences 908.1 (2000): 143-154.

9. Mah LY and KM Ryan. "Autophagy and Cancer". Cold Spring Harbor Perspectives in Biology 4.1 (2012): a008821.

10. Wani WY., et al. "O-GlcNAc regulation of autophagy and $\alpha$-synuclein homeostasis; implications for Parkinson's disease". Molecular Brain 10 (2017): 32.

11. Xilouri M., et al. "Autophagy and Alpha-Synuclein: Relevance to Parkinson's Disease and Related Synucleopathies". Movement Disorders 31.2 (2016): 178-192.

12. Bang Y., et al. "Preconditioning stimulus of proteasome inhibitor enhances aggresome formation and autophagy in differentiated SH-SY5Y cells". Neuroscience Letters 566 (2014): 263268.

13. Regitz C., et al. "Resveratrol reduces amyloid-beta (Aß1-42)induced paralysis through targeting proteostasis in an Alzheimer model of Caenorhabditis elegans". European Journal of Nutrition 55.2 (2016): 741-747.

14. Xu P., et al. "Triptolide Inhibited Cytotoxicity of Differentiated PC12 Cells Induced by Amyloid-Beta (25-35) via the Autophagy Pathway". PLoS ONE 10.11 (2015): e0142719. 
15. Ross CA and SJ Tabrizi. "Huntington's disease: from molecular pathogenesis to clinical treatment". The Lancet Neurology 10.1 (2011): 83-98.

16. Cook C and L Petrucelli. "A critical evaluation of the ubiquitin-proteasome system in Parkinson's disease". Biochimica et Biophysica Acta (BBA) - Molecular Basis of Disease 1792.7 (2009): 664-675.

17. Gadhave K., et al. "The ubiquitin proteasomal system: a potential target for the management of Alzheimer's disease". Journal of Cellular and Molecular Medicine 20.7 (2016): 1392-1407.

18. Yoshida H. "ER stress and diseases". FEBS Journal 274.3 (2007): 630-658.

19. Szegezdi E., et al. "Mediators of endoplasmic reticulum stressinduced apoptosis”. EMBO Reports 7.9 (2006): 880-885.

20. Ron D and P Walter. "Signal integration in the endoplasmic reticulum unfolded protein response". Nature Reviews Molecular Cell Biology 8.7 (2007): 519-529.

21. Ustundag Y., et al. "Proteasome inhibition-induces endoplasmic reticulum dysfunction and cell death of human cholangiocarcinoma cells". World Journal of Gastroenterology : WJG 13.6 (2007): 851-857.

22. Cheng B., et al. "Insulin-like Growth Factor-I Mediates Neuroprotection in Proteasome Inhibition-Induced Cytotoxicity in SH-SY5Y Cells". Molecular and Cellular Neurosciences 47.3 (2011): 181-190.

23. Cheng B., et al. "Retinoic acid protects against proteasome inhibition associated cell death in SH-SY5Y cells via the AKT pathway". Neurochemistry International 62.1 (2013): 31-42.

24. Gies E., et al. "Niclosamide Prevents the Formation of Large Ubiquitin-Containing Aggregates Caused by Proteasome Inhibition". PLoS ONE 5.12 (2010): e14410.

25. Bhattacharyya J., et al. "Niclosamide-conjugated polypeptide nanoparticles inhibit Wnt signaling and colon cancer growth". Nanoscale (2017).

26. Arend RC., et al. "Niclosamide and its analogs are potent inhibitors of Wnt/ $\beta$-catenin, mTOR and STAT3 signaling in ovarian cancer". Oncotarget 7.52 (2016): 86803-86815.

27. You S., et al. "Disruption of STAT3 by niclosamide reverses radioresistance of human lung cancer". Molecular Cancer Therapeutics 13.3 (2014): 606-616.

28. Ren X., et al. "Identification of Niclosamide as a New SmallMolecule Inhibitor of the STAT3 Signaling Pathway". ACS Medicinal Chemistry Letters 1.9 (2010): 454-459.
29. Tao H., et al. "Niclosamide ethanolamine-induced mild mitochondrial uncoupling improves diabetic symptoms in mice". $\mathrm{Na}$ ture Medicine 20.11 (2014): 1263-1269.

30. Han P., et al. "Niclosamide ethanolamine improves diabetes and diabetic kidney disease in mice". American Journal of Translational Research 10.4 (2018): 1071-1084.

31. Cerles O., et al. "Niclosamide Inhibits Oxaliplatin Neurotoxicity while Improving Colorectal Cancer Therapeutic Response". Molecular Cancer Therapeutics 16.2 (2017): 300.

32. Zhang LX., et al. "Niclosamide attenuates inflammatory cytokines via the autophagy pathway leading to improved outcomes in renal ischemia/reperfusion injury". Molecular Medicine Reports 16.2 (2017): 1810-1816.

33. Cheng B., et al. "Niclosamide induces protein ubiquitination and inhibits multiple pro-survival signaling pathways in the human glioblastoma U-87 MG cell line". PloS one 12.7 (2017): e0184324e0184324.

34. Park HS., et al. "Proteasome inhibitor MG132-induced apoptosis via ER stress-mediated apoptotic pathway and its potentiation by protein tyrosine kinase p56lck in human Jurkat T cells". Biochemical Pharmacology 82.9 (2011): 1110-1125.

35. Nguyen H and BD Uhal. "The unfolded protein response controls ER stress-induced apoptosis of lung epithelial cells through angiotensin generation". American Journal of Physiology - Lung Cellular and Molecular Physiology 311.5 (2016): L846.

36. Liu XA., et al. "Expression of the hyperphosphorylated tau attenuates ER stress-induced apoptosis with upregulation of unfolded protein response". Apoptosis 17.10 (2012): 1039-1049.

37. Pandey UB., et al. "HDAC6 rescues neurodegeneration and provides an essential link between autophagy and the UPS". Nature 447.7146 (2017): 860-864.

38. Cheng B., et al. "N-Acetylcysteine in Combination with IGF-1 Enhances Neuroprotection against Proteasome DysfunctionInduced Neurotoxicity in SH-SY5Y Cells". Parkinson's Disease (2016): 6564212.

39. Ding WX., et al. "Linking of Autophagy to Ubiquitin-Proteasome System Is Important for the Regulation of Endoplasmic Reticulum Stress and Cell Viability". The American Journal of Pathology 171.2 (2007): 513-524.

40. Marciniak SJ., et al. "CHOP induces death by promoting protein synthesis and oxidation in the stressed endoplasmic reticulum". Genes and Development 18.24 (2004): 3066-3077.

41. Kaufmann SH., et al. "Specific Proteolytic Cleavage of Poly (ADPribose) Polymerase: An Early Marker of Chemotherapy-induced Apoptosis". Cancer Research 53.7 (1993): 3976-3985. 
42. Harhouri K., et al. "MG132-induced progerin clearance is mediated by autophagy activation and splicing regulation". EMBO Molecular Medicine 9.9 (2017): 1294-1313.

43. Marino G., et al. "Autophagy for tissue homeostasis and neuroprotection". Current Opinion in Cell Biology 23.2 (2011): 198206.

44. Sánchez-Pérez AM., et al. "Parkinson's Disease and Autophagy”. Parkinson's Disease (2012): 429524.

45. Ouyang, L., et al. "Programmed cell death pathways in cancer: a review of apoptosis, autophagy and programmed necrosis". Cell Prolif 45.6 (2012): 487-498.

46. Tan CC., et al. "Autophagy in aging and neurodegenerative diseases: implications for pathogenesis and therapy". Neurobiology Aging 35.5 (2014): 941-957.

47. Mariño G and C López-Otín. "Autophagy: molecular mechanisms, physiological functions and relevance in human pathology". Cellular and Molecular Life Sciences CMLS 61.12 (2004): 1439-1454.

48. Zhang Y., et al. "The role of ubiquitin proteasomal system and autophagy-lysosome pathway in Alzheimer's disease". Reviews in the Neurosciences (2017).

49. Yuan J., et al. "Diversity in the Mechanisms of Neuronal Cell Death". Neuron 40.2 (2003): 401-413.

\section{Assets from publication with us}

- Prompt Acknowledgement after receiving the article

- Thorough Double blinded peer review

- Rapid Publication

- Issue of Publication Certificate

- High visibility of your Published work

Website: www.actascientific.com/

Submit Article: www.actascientific.com/submission.php

Email us: editor@actascientific.com

Contact us: +919182824667 